\title{
Fractal Properties of Critical Invariant Curves
}

\author{
Brian R. Hunt ${ }^{1}$, Konstantin M. Khanin ${ }^{2,3}$, \\ Yakov G. Sinai ${ }^{2,3}$, and James A. Yorke ${ }^{1}$
}

December 31, 1995

\begin{abstract}
We examine the dimension of the invariant measure for some singular circle homeomorphisms for a variety of rotation numbers, through both the thermodynamic formalism and numerical computation. The maps we consider include those induced by the action of the standard map on an invariant curve at the critical parameter value beyond which the curve is destroyed. Our results indicate that the dimension is universal for a given type of singularity and rotation number, and that among all rotation numbers, the golden mean produces the largest dimension.

Keywords: thermodynamic formalism, fractal dimension, invariant measure, circle homeomorphism, rotation number, twist map, critical curve, renormalization.
\end{abstract}

\section{Introduction}

In the last decade there has been much interest in the study of transitions to chaos and the onset of stochasticity in dynamical systems. It has been discovered that fractal structures often appear on the border between regular and chaotic dynamical behavior. In the best known examples the fractal objects exhibit robust scaling properties and renormalization ideas can be naturally applied. This was first understood by M. Feigenbaum for the perioddoubling route to chaos - a cascade of period-doubling bifurcations accumulating to a critical parameter corresponding to a Cantor set attractor with universal scaling properties [7, 8]. Renormalization ideas also have been used to study the transition to chaos in two different

\footnotetext{
${ }^{1}$ Institute for Physical Science and Technology, University of Maryland, College Park, MD 20742. These authors were supported by the National Science Foundation (Divisions of Mathematical and Physical Sciences) and by the U.S. Department of Energy (Offices of Scientific Computing and Energy Research).

${ }^{2}$ Department of Mathematics, Princeton University, Princeton, NJ 08544-1000.

${ }^{3}$ Landau Institute of Theoretical Physics, Russian Academy of Sciences, Moscow 117334, Russia.
} 
situations which we examine below in this paper. One of them comes from the disappearance of quasiperiodic motions in the case of critical circle maps [9, 23]. The other is connected with the destruction of invariant tori for two degree of freedom Hamiltonian systems [10, 21]. This case can be reduced, by considering a Poincaré return map, to studying the destruction of invariant curves for area-preserving maps of the cylinder. In this paper we will study the fractal properties of the dynamics in both situations.

Consider first the case of critical circle maps. A familiar example is the two-parameter family of maps

$$
x \mapsto g_{k, \omega}(x)=x+\omega-\frac{k}{2 \pi} \sin 2 \pi x(\bmod 1), \quad k \geq 0 .
$$

For $0 \leq k<1$ this is a family of diffeomorphisms of the circle. When $k=1$ the maps are still homeomorphisms, but there is a cubic inflection point at $x=0$, and hence the inverse of the map is not differentiable. For $k>1$ the maps are no longer invertible.

For a circle homeomorphism $g$ we can define the "rotation number" of $g$ to be, roughly speaking, the average amount $x$ is rotated around the circle per iterate of the map. More precisely, the rotation number $\rho$ associated with $g$ is defined by "lifting" $g$ to a map from the real line to itself which, when taken modulo one, gives the action of $g$ on the circle. On the real line $g$ is a strictly increasing function which satisfies $g(x+1)=g(x)+1$. Let $g^{n}$ denote the $n$th iterate of $g$, and let

$$
\rho=\lim _{n \rightarrow \infty} \frac{g^{n}(x)}{n}
$$

the limit is independent of the initial point $x$. (Strictly speaking, the lift of $g$ to the real line is only determined up to an additive integer, so the same is true for $\rho$; we use the convention that $0 \leq \rho<1$.)

If the rotation number $\rho$ of a circle homeomorphism $g$ is rational, say $\rho=p / q$, then all trajectories converge to some periodic orbit which makes $p$ rotations every $q$ iterates. Irrational values of $\rho$ correspond to quasiperiodic motion and the dynamics is topologically conjugate to a rigid rotation by the angle $\rho$. It is found that for a fixed value $k \in[0,1]$, there exists an interval of values of the parameter $\omega$ for which $g_{k, \omega}$ has a given rational rotation number, and a unique value of $\omega$, say $\omega_{k}(\rho)$, corresponding to each irrational rotation number $\rho$. For $k<1$ it follows from the theorem of M. Herman [14] that that the Cantor set of $\omega$ with irrational rotation numbers has positive Lebesgue measure, while it has been shown by G. Świątek [27] that this set has Lebesgue measure zero for $k=1$. Moreover, it was shown for $k=1$ that the Hausdorff dimension of this set is less than 1 ; see $[18,11]$. For $k>1$ the map $g_{k, \omega}$ is no longer a homeomorphism and different trajectories of the map may have different rotation numbers; the dynamics thus becomes more complicated.

In addition to the fractal structure of parameter space in this example, we find that orbit structure in phase space becomes fractal when $k=1$. One of the main purposes of our 
numerical study is to show that for irrational rotation numbers, every trajectory, though dense in the circle, is concentrated on a fractal set in the following sense. It is well known that circle homeomorphisms are uniquely ergodic, that is there exists a unique invariant probability measure $\mu$, so that every trajectory is asymptotically distributed according to $\mu$. The invariant measure $\mu$ is absolutely continuous with respect to Lebesgue measure for $0 \leq k<1$ and for Diophantine rotation numbers $\rho$, that is, such numbers $\rho$ that for every $\varepsilon>0$ there exists a $C>0$ such that for all integers $p$ and $q>0$,

$$
\left|\rho-\frac{p}{q}\right|>\frac{C}{q^{2+\varepsilon}} .
$$

The set of Diophantine numbers has full Lebesgue measure. However, when $k=1$, for all irrational rotations numbers the invariant measure $\mu$ is singular with respect to Lebesgue measure, and furthermore has Hausdorff dimension (described below) less than 1 [18, 12, 26].

For $k=1$ and various irrational rotation numbers, we study the fractal dimension of $\mu$, which can be defined in several ways (see for instance [6]). One definition is based on the Hausdorff dimension for sets. We say that the Hausdorff dimension of a probability measure $\mu$ is

$$
d_{H}(\mu)=\inf _{\{S \subset T: \mu(S)=1\}} d_{H}(S)
$$

what $d_{H}(S)$ denotes the Hausdorff dimension of the set $S$. Thus if $d_{H}(\mu)<1$, then we can say that trajectories asymptotically spend "almost all" of their time on a set of Hausdorff dimension less than one. Another notion of dimension for measures is the "information dimension", which we recall in Section 2. These dimensions are generally expected to coincide for the natural invariant measures of dynamical systems; see [6]. Other fractal properties of the invariant measure are captured in a graph of an " $f(\alpha)$ curve"; see [13] for an example involving the map $g_{k, \omega}$ for a particular rotation number.

By the principles of renormalization we expect that the dimension of the invariant measure should depend only on the rotation number and the nature of the singularity which makes the map critical, but not on the particular family of maps. The family $g_{k, \omega}$ illustrates the case of a cubic singularity. We will consider also the case of a "break singularity", that is a discontinuity of the derivative, as exemplified by the family of maps

$$
x \mapsto h_{c, \omega}(x)=\frac{x}{c+(1-c) x}+\omega(\bmod 1), \quad c>0
$$

The first derivative of this homeomorphism has a jump discontinuity at $x=0$, and the ratio of the left-hand derivative to right-hand derivative is $c^{2}$. This ratio characterizes the break singularity in the same sense as the order of the inflection point characterized the singularity in the previous example. For all $c \neq 1$ the set of $\omega$ which have irrational rotation numbers has 
Lebesgue measure zero [20]. It can be shown also that the invariant measures are singular for every irrational rotation number.

Again an important question is how the dimension of the invariant measure depends on the rotation number and the value of $c$. As above, renormalization group ideology suggests that the dimensions we calculate to be universal, in the sense that every homeomorphism with the same rotation number and same type of break singularity should have an invariant measure of the same dimension [19].

Consider next the case of area-preserving diffeomorphisms of the cylinder which are orientation-preserving and have zero Calabi invariant (the last condition means that there is no aggregate drift along the axial direction of the cylinder). These include maps of the form

$$
\begin{aligned}
& x \mapsto x+y-k f(x)(\bmod 1) \\
& y \mapsto y-k f(x)
\end{aligned}
$$

where $f(x)$ is an analytic, periodic function with period 1 and $\int_{0}^{1} f(x) d x=0$. When $f(x)=$ $(1 / 2 \pi) \sin 2 \pi x$, this map is the well-known "standard map"; see for example [24]. For $k=0$, the phase space decomposes into invariant circles $y=\rho$ with every possible rotation number $\rho$. For positive $k$, the map induces a homeomorphism on every invariant curve surrounding the circle, and thus every invariant curve has a rotation number associated with it. For a given irrational rotation number $\rho$ which is typical (in the sense of Diophantine), KAM theory guarantees that for small $k$ there is and invariant curve with rotation number $\rho$, and this curve is analytic. As $k$ increases, the curve with rotation number $\rho$ is eventually destroyed and does not exist for $k$ greater than some critical parameter value $k_{\rho}$.

Though the critical invariant curve is not a fractal, the dynamics on the invariant curve is concentrated on a fractal set in the same sense as above. The map restricted to the curve is a homeomorphism and thus induces a unique invariant probability measure $\mu$ supported on the curve. It has been expected that $\mu$ is fractal for critical invariant curves; see [22] for graphs of $f(\alpha)$ curves for $\mu$ for a few rotation numbers. The dimension of $\mu$ is nonetheless difficult to distinguish from 1 ; in this paper we give strong numerical evidence that the dimension is in fact strictly less than 1 for a wide variety of rotation numbers.

Critical invariant curves, in contrast with KAM curves, are not analytic. They are known to be at least Lipschitz [4, 15], but not $C^{2+\varepsilon}$ for any $\varepsilon>0$ [17]. Our numerical results for the dimension of the invariant measure indicate that the smoothness can be less than $C^{2}$, since for $C^{2}$ diffeomorphisms and the rotation numbers we study, the invariant measure is absolutely continuous [16]. Indeed, in the case when the rotation number is the golden mean, the maximum smoothness of the critical curve has been shown to be $C^{1+\gamma}$ where $\gamma<1$ [25].

Our numerical results are based on both a direct approximation of the invariant measure and its information dimension, and a computation of the Hausdorff dimension of the measure 
based on the thermodynamic formalism. The thermodynamic formalism has been used widely in the context of renormalization starting with [28]; see also [2, 5, 1].

In Section 2 we describe in more detail both approaches, and in Section 3 we present our numerical results for a variety of rotations numbers. Some concluding remarks are given in Section 4.

\section{Methods}

Let $f$ be a homeomorphism on the circle and let $S$ be the circle. All of our computations are based on forming partitions of $S$ using the points of a trajectory $x_{0}, x_{1}, x_{2}, \ldots, x_{N-1}$ of $f$. The numbers $N$ of intervals in the partitions we study are based on the continued fraction expansion of the rotation number $\rho$ of $f$. This expansion is described in the following paragraph.

We write $\rho=\left[a_{1}, a_{2}, a_{3}, \ldots\right]$ where $a_{1}, a_{2}, \ldots$ are positive integers and

$$
\rho=\frac{1}{a_{1}+\frac{1}{a_{2}+\frac{1}{a_{3}+\cdots}}} .
$$

The numbers $a_{1}, a_{2}, \ldots$ which make up the continued fraction for $\rho$ can be generated recursively by letting $t_{0}=\rho$, and for $n \geq 1$ letting $\left(a_{n}, t_{n}\right)$ be the integral and fractional parts of $1 / t_{n-1}$. (If $\rho$ is rational this procedure eventually terminates when $t_{n}=0$ for some $n$, but if $\rho$ is irrational an infinite sequence of $a_{n}$ will be generated.) We define $p_{n}$ and $q_{n}$ to be the numerator and denominator of the rational approximation to $\rho$ given by truncating the continued fraction at $a_{n}$, that is

$$
\frac{p_{n}}{q_{n}}=\frac{1}{a_{1}+\frac{1}{a_{2}+\cdots+\frac{1}{a_{n}}}} .
$$

(Recursively, $p_{n}=a_{n} p_{n-1}+p_{n-2}$ and $q_{n}=a_{n} q_{n-1}+q_{n-2}$ for $n \geq 2$ with $p_{0}=0, q_{0}=1$, $p_{1}=1, q_{1}=a_{1}$.) The ratio $p_{n} / q_{n}$ is called the $n$th "convergent" of $\rho$.

For $n \geq 1$ we consider the partition $\mathcal{P}_{n}$ generated by the trajectory $x_{0}, x_{1}, \ldots, x_{q_{n}+q_{n-1}-1}$. Through these first $q_{n}+q_{n-1}$ iterates, the closest points of the trajectory to $x_{0}$ will be $x_{q_{n-1}}$ on one side and $x_{q_{n}}$ on the other side. Thus the intervals of $\mathcal{P}_{n}$ can be thought of as the first $q_{n}$ iterates of the interval $I_{n-1}$ bounded by $x_{0}$ and $x_{q_{n-1}}$ and the first $q_{n-1}$ iterates of the interval $I_{n}$ bounded by $x_{0}$ and $x_{q_{n}}$; see [24].

We study mainly those rotation numbers with eventually periodic continued fractions. The reason for this is twofold. First, our dimension computations are of course based on 
a finite level of precision, and typically we can only study the partitions $\mathcal{P}_{n}$ for relatively small $n$. Thus in order for our estimates to be reliable, it is important for the initial terms in the continued fraction expansion $\rho=\left[a_{1}, a_{2}, \ldots\right]$ to be characteristic of the entire expansion. Furthermore, if the continued fraction (eventually) has period $j$, the partitions $\mathcal{P}_{n}$ and $\mathcal{P}_{n+j}$ will be structurally similar, making comparisons between the two especially robust. The second reason for studying periodic continued fractions is that those are the rotation numbers whose invariant curves are most readily observable for planar maps like the standard map.

\subsection{Information Dimension}

Given a partition of the circle $S$ into intervals $\left\{J_{m}\right\}$, form the quotient

$$
\frac{\sum_{m} \mu\left(J_{m}\right) \log \mu\left(J_{m}\right)}{\sum_{m} \mu\left(J_{m}\right) \log \left|J_{m}\right|},
$$

where $\left|J_{m}\right|$ denotes the length of the interval $J_{m}$ and $\mu$ is the natural invariant measure associated with the map $f$. As the size of the intervals in the partition approaches zero, this ratio generally approaches a limit which we call the information dimension [3] of $\mu$, denoted $d_{I}(\mu)$.

For the partitions $\mathcal{P}_{n}$ described above, the measure $\mu$ of each interval is known exactly. Because $f$ is conjugate to a rigid rotation by $\rho$, and the invariant measure for the latter map is just Lebesgue measure, the measure of the interval between $x_{0}$ and $x_{q_{n}}$ is $\left|\rho q_{n}-p_{n}\right|$. Thus we can estimate the information dimension of the critical invariant curve with rotation number $\rho$ by estimating the parameter value which gives rise to this curve, computing a trajectory of length $q_{n}+q_{n-1}$, and measuring the lengths of the intervals which make up the partition $\mathcal{P}_{n}$. For the circle maps the calculations are similar, except that we need not search for the critical parameter value.

\subsection{The Thermodynamic Formalism}

Now we construct the symbolic representation for the system of partitions $\mathcal{P}_{n}$. Namely, each interval of the partition $\mathcal{P}_{n}$ we'll label with a word $\left(\sigma_{1}, \sigma_{2}, \ldots, \sigma_{n}\right)$ of length $n$ in some finite alphabet. Each $\sigma_{i}$ takes values in a finite alphabet depending on $i: \sigma_{i}=0,1,2, \ldots, a_{i}$ and $P$. Here $a_{i}$ is an integer entry in continued fraction expansion for $\rho$ and $P$ is a special letter whose meaning will be explained shortly. The basic property one needs for correct symbolic representation is the following: if $\triangle(n+1)$ is an interval which is an element of the partition $\mathcal{P}_{n+1}$ and $\triangle(n)$ is an element of $\mathcal{P}_{n}$ containing $\triangle(n+1)$, then the first $n$ letters of the word for $\triangle(n+1)$ coincide with the word for $\triangle(n)$. We just add one more letter depending on the position of $\triangle(n+1)$ inside $\triangle(n)$. We add the special letter $P$ if $\triangle(n+1)$ coincides with 
$\triangle(n)$, and add letters $0,1, \ldots, a_{n}$ if $\triangle(n)$ is partitioned into $\left(a_{n}+1\right)$ parts. It can be seen that there are only these two possibilities. The intervals which are generated by $\left(x_{0}, x_{q_{n-1}}\right)$ are those which are partitioned when we pass from $\mathcal{P}_{n}$ to $\mathcal{P}_{n+1}$, and we add letter 0 to the subinterval closest to the point $x_{0}$, letter 1 to the next subinterval, and so on. We do the same for the images of $\left(x_{0}, x_{q_{n-1}}\right)$ : for $\left(x_{i}, x_{i+q_{n-1}}\right)$ we add letter 0 to the subinterval closest to $x_{i}$, letter 1 to the next one, and so on. This procedure defines the symbolic representation inductively. The letter $P$ (for "preserve") plays a special role. It can be seen that we always have $P$ after 0 , and always have 0 before $P$. One could avoid this letter but then the words corresponding to different intervals of partition $\mathcal{P}_{n}$ would have different length.

The system of partitions $\mathcal{P}_{n}, n \geq 1$ and our system of labeling generates the symbolic representation. Namely, there is a correspondence between the points of the circle and the infinite sequences of symbols $\left(\sigma_{1}, \sigma_{2}, \ldots, \sigma_{n}, \ldots\right)$. In ergodic theory such sequences are called subshifts of finite type. This correspondence is one-to-one except for the countable set of endpoints of the partitions $\mathcal{P}_{n}, n \geq 1$. Every measure on the circle generates a corresponding measure on the infinite words $\left(\sigma_{1}, \sigma_{2}, \ldots, \sigma_{n}, \ldots\right)$. Denote by $\operatorname{Pr}$ the measure generated by Lebesgue measure. The thermodynamic formalism deals with this measure, or random process, $\operatorname{Pr}$. From the point of view of statistical mechanics $\operatorname{Pr}$ is a one-dimensional lattice system, and $\sigma_{i}$ is a spin variable sitting on a lattice point $i$ and taking values $P, 0,1,2, \ldots$, . Denote the conditional probability of a spin $\sigma_{n}$ under the condition of fixed $\sigma_{n-1}, \sigma_{n-2}, \ldots, \sigma_{1}$ by

$$
\operatorname{Pr}\left(\sigma_{n} \mid \sigma_{n-1}, \ldots, \sigma_{1}\right)=\frac{\left|\triangle\left(\sigma_{1}, \ldots, \sigma_{n-1}\right)\right|}{\left|\triangle\left(\sigma_{1}, \ldots, \sigma_{n}\right)\right|}
$$

where $\triangle\left(\sigma_{1}, \ldots, \sigma_{n}\right), \triangle\left(\sigma_{1}, \ldots, \sigma_{n-1}\right)$ are corresponding intervals of partitions $\mathcal{P}_{n}$ and $\mathcal{P}_{n-1}$ respectively, $\triangle\left(\sigma_{1}, \ldots, \sigma_{n}\right) \subset \triangle\left(\sigma_{1}, \ldots, \sigma_{n-1}\right)$. Write

$$
U_{n}\left(\sigma^{(1)}=\sigma_{n}, \sigma^{(2)}=\sigma_{n-1}, \ldots, \sigma^{(n)}=\sigma_{1}\right) \equiv U_{n}\left(\sigma^{(1)}, \ldots, \sigma^{(n)}\right) \equiv \log \operatorname{Pr}\left(\sigma_{n} \mid \sigma_{n-1}, \ldots, \sigma_{1}\right) .
$$

We shall call $U_{n}$ the potential at point $n$. It has the meaning of the energy of interaction of the spin $\sigma_{n}$ with all the spins $\left(\sigma_{n-1}, \ldots, \sigma_{1}\right)$. Notice that the order of variables in $U_{n}$ is inverted. The potentials $U_{n}$ have the following key property: they depend mostly on the first variables $\sigma^{(1)}, \sigma^{(2)}, \ldots$ and exponentially weakly depend on the last variables $\sigma^{(n)}, \sigma^{(n-1)}, \ldots$. This fact is based on numerical evidence in the case of critical invariant curves and can be rigorously proved for critical circle maps [18] and for circle maps with a break singularity [20]. And this is the crucial property for the thermodynamic approach. More precisely it means the following. Suppose we have two sequences $\left(\sigma^{(1)}, \ldots, \sigma^{(n)}\right)$ and $\left(\varepsilon^{(1)}, \ldots, \varepsilon^{(n)}\right)$, and $\sigma^{(1)}=\varepsilon^{(1)}, \ldots, \sigma^{(k)}=\varepsilon^{(k)}$. Then

$$
\left|U_{n}\left(\sigma^{(1)}, \ldots, \sigma^{(n)}\right)-U_{n}\left(\varepsilon^{(1)}, \ldots, \varepsilon^{(n)}\right)\right|<C \lambda^{k},
$$


where $\lambda<1$ does not depend on $n$. This means that the system of statistical mechanics corresponding to $U_{n}$ is nice: there are no phase transitions, correlations decay exponentially, and the process $\operatorname{Pr}$ is "almost" Markovian. Since

$$
\left|\triangle\left(\sigma_{1}, \ldots, \sigma_{n}\right)\right|=\exp \left\{U_{n}\left(\sigma_{n}, \ldots, \sigma_{1}\right)+U_{n-1}\left(\sigma_{n-1}, \ldots, \sigma_{1}\right)+\ldots+U_{2}\left(\sigma_{2}, \sigma_{1}\right)+U_{1}\left(\sigma_{1}\right)\right\}
$$

the partition function

$$
Z_{n}(\beta)=\sum_{\left(\sigma_{1}, \ldots, \sigma_{n}\right)}\left|\triangle\left(\sigma_{1}, \ldots, \sigma_{n}\right)\right|^{\beta}
$$

coincides up to a bounded constant factor with the partition function for our system of statistical mechanics.

Denote by $P_{G}(\beta)$ the Gibbs measure on the space of sequences $\left\{\left(\sigma_{1}, \sigma_{2}, \ldots\right)\right\}$ corresponding to the potentials $U_{n}$ and the "inverse temperature" $\beta$. Notice that $\beta=0,1$ correspond to the invariant measure $\mu$ on the circle and Lebesgue measure, respectively. In other words, $P_{G}(0)$ is equal to the measure generated by $\mu$ and $P_{G}(1)$ coincided with $\mathrm{Pr}$. All information about dimension can be extracted from the free energy $f_{n}(\beta)=(1 / n) \log Z_{n}(\beta)$. The Hausdorff dimension of the measure $\mu$ is given by

$$
\operatorname{dim}_{H}(\mu)=\lim _{n \rightarrow \infty} \frac{f_{n}(0)}{f_{n}^{\prime}(0)} .
$$

The reason for this formula is as follows. It is not hard to show that the above limit is equal to the information dimension defined previously, and the applicability of the thermodynamic formalism guarantees that both dimensions are the same [29].

It is generally believed that in the case of rotation numbers with periodic continued fraction expansions, the potentials $U_{n}$ tend to a limit when $n \rightarrow \infty$, and this limit is just $s$ different limiting potentials, where $s$ is a period of the continued fraction expansion. It means that there are $s$ functions $U_{0}, \ldots, U_{s-1}$ (all depend on infinite sequences) such that

$$
\begin{gathered}
U_{n s}\left(\sigma^{(1)}, \ldots, \sigma^{(n)}\right) \rightarrow U_{0}, \quad n \rightarrow \infty \\
U_{n s+1}\left(\sigma^{(1)}, \ldots, \sigma^{(n s+1)}\right) \rightarrow U_{1}, \quad n \rightarrow \infty \\
U_{n s+s-1}\left(\sigma^{(1)}, \ldots, \sigma^{(n s+(s-1))}\right) \rightarrow U_{s-1}, \quad n \rightarrow \infty .
\end{gathered}
$$

Thus the limiting system of statistical mechanics has periodic potential $\left(U_{0}, U_{1}, \ldots, U_{s-1}\right)$. The next step is to form the transfer-matrix operator $\mathcal{L}(\beta)=\mathcal{L}_{s-1}(\beta) \cdot \ldots \cdot \mathcal{L}_{1}(\beta) \cdot \mathcal{L}_{0}(\beta)$, where the $\mathcal{L}_{i}(\beta)$ are transfer-matrix operators corresponding to potentials $U_{i}, 0 \leq i \leq s-1$ :

$$
\left(\mathcal{L}_{i}(\beta) f\right)\left(\sigma^{(1)}, \sigma^{(2)}, \ldots, \sigma^{(n)}, \ldots\right)=\sum_{\sigma=P, 0,1, \ldots, a_{i}} e^{U_{i}\left(\sigma, \sigma^{(1)}, \sigma^{(2)}, \ldots, \sigma^{(n)}\right)} f\left(\sigma, \sigma^{(1)}, \sigma^{(2)}, \ldots, \sigma^{(n)}, \ldots\right)
$$


Denote by $\lambda(\beta)$ the largest eigenvalue of the linear operator $\mathcal{L}(\beta)$. Then the partition function $Z_{n}(\beta)$ behaves like $\left(\lambda(\beta)^{\frac{n}{s}}\right.$. It follows that the free energy

$$
f_{n}(\beta)=\frac{\log Z_{n}(\beta)}{n} \rightarrow \frac{\log \lambda(\beta)}{s} \quad \text { as } \quad n \rightarrow \infty .
$$

In real calculations one should use an approximation of infinite-dimensional operator $\mathcal{L}(\beta)$ with finite matrices. This means that we approximate the potentials $U_{0}, U_{1}, \ldots, U_{s-1}$ by potentials depending on the first $k$ symbols only and restrict the action of $\mathcal{L}_{i}(\beta), i=$ $0, \ldots, s-1$ to the subspaces of functions depending on $k-1$ symbols. This approximation is exact in the limit $k \rightarrow \infty$. Let us consider in more detail the approximation scheme in the simplest case of "golden mean" $\rho=\frac{\sqrt{5}-1}{2}$. In this case there is only one limiting potential which we shall denote by $U\left(\sigma^{(1)}, \ldots, \sigma^{(n)}, \ldots\right)$.

Fix some $k$ and consider intervals of the partition $\mathcal{P}_{n}$ inside the intervals $\left(x_{q_{n-k-1}}, x_{0}\right)$, $\left(x_{0}, x_{q_{n-k}}\right)$. For $k=2$ we have the following picture.

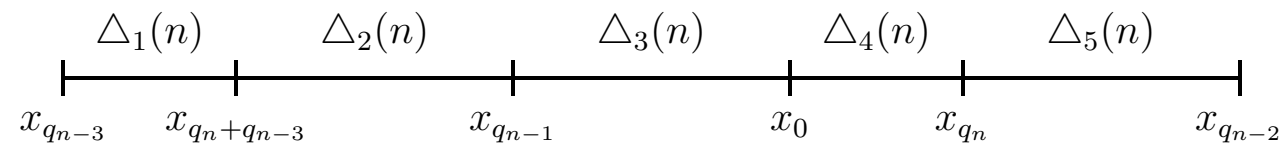

The symbolic coordinates of the intervals $\triangle_{1}(n), \ldots, \triangle_{5}(n)$ are

$$
\begin{gathered}
\triangle_{1}(n)=(\ldots, P, 1,0), \quad \triangle_{2}(n)=(\ldots, P, 1,1), \quad \triangle_{3}(n)=(\ldots, P, 0, P), \\
\triangle_{4}(n)=(\ldots, 0, P, 0), \quad \triangle_{5}(n)=(\ldots, 0, P, 1) .
\end{gathered}
$$

For $k=2$ we use an approximation of $U_{n}$ by a function depending on two symbols:

$$
\begin{gathered}
e^{U_{n}(0,1)}=\frac{\triangle_{1}(n)}{\triangle_{1}(n)+\triangle_{2}(n)} \rightarrow \gamma, \quad e^{U_{n}(1,1)}=\frac{\triangle_{2}(n)}{\triangle_{2}(n)+\triangle_{2}(n)} \rightarrow 1-\gamma, \quad n \rightarrow \infty \\
e^{U_{n}(P, 0)}=\frac{\triangle_{3}(n)}{\triangle_{3}(n)}=1 \\
e^{U_{n}(0, P)}=\frac{\triangle_{4}(n)}{\triangle_{4}(n)+\triangle_{5}(n)} \rightarrow \alpha, \quad e^{U_{n}(1, P)}=\frac{\triangle_{5}(n)}{\triangle_{4}(n)+\triangle_{5}(n)} \rightarrow 1-\alpha, \quad n \rightarrow \infty .
\end{gathered}
$$

Consider the restriction of the action of $\mathcal{L}(\beta)$ to the subspace of functions depending on one symbol only. Then in the limit $n \rightarrow \infty$ we obtain the following approximation for the transfer-matrix operator $\mathcal{L}(\beta)$ :

$$
\mathcal{L}^{(2)}(\beta)=\left(\begin{array}{ccc}
0 & 0 & 1 \\
\gamma^{\beta} & (1-\gamma)^{\beta} & 0 \\
\alpha^{\beta} & (1-\alpha)^{\beta} & 0
\end{array}\right) .
$$


The first column (and row) corresponds to $f(0)$, the second to $f(1)$, third to $f(P)$. The values of $\alpha$ and $\gamma$ generally must be obtained from numerical data. For the standard map in the critical case we find that $\alpha \approx 0.4996, \gamma \approx 0.3543$. Denote the largest eigenvalue of $\mathcal{L}(\beta)$ by $\lambda(\beta)$. Then $f_{n}(\beta) \rightarrow \log (\lambda(\beta)), n \rightarrow \infty$. One can check that $\lambda(0)=1 / \rho, \lambda(1)=1$. The Hausdorff dimension of the invariant measure $\mu$ is then given by

$$
d_{H}(\mu)=\frac{-\log (\lambda(0))}{\left.\log (\lambda(\beta))^{\prime}\right|_{\beta=0}}=\frac{\log \rho}{\rho \lambda^{\prime}(0)} .
$$

Using the approximation of $\mathcal{L}(\beta)$ by $\mathcal{L}^{(2)}(\beta)$ it is easy to show that

$$
\lambda^{(2) \prime}(0)=\frac{\log (1-\gamma)+(1-\rho) \log \alpha+\rho \log (\gamma(1-\alpha))}{2 \rho+1},
$$

which leads to the following approximate dimension:

$$
d_{H}^{(2)}(\mu)=\frac{(\log \rho)(2 \rho+1)}{\rho \log (1-\gamma)+\rho(1-\rho) \log \alpha+\rho^{2} \log (\gamma(1-\alpha))} .
$$

For the standard map we obtain

$$
d_{H}^{(2)}(\mu) \approx 0.9827
$$

The next approximation corresponds to $k=3$ and the following picture.

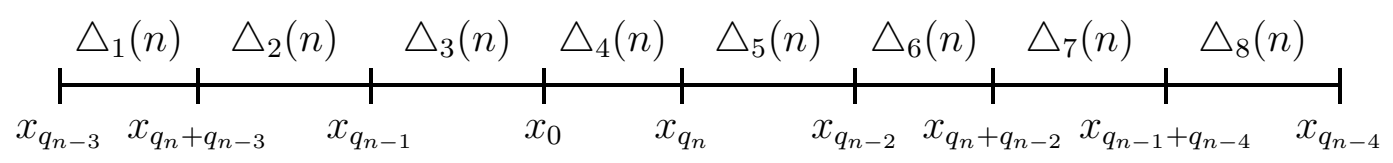

Here

$$
\begin{gathered}
\triangle_{1}(n)=(\ldots, 0, P, 1,0), \quad \triangle_{2}(n)=(\ldots, 0, P, 1,1), \quad \triangle_{3}(n)=(\ldots, 0, P, 0, P), \\
\triangle_{4}(n)=(\ldots, P, 0, P, 0), \quad \triangle_{5}(n)=(\ldots, P, 0, P, 1), \\
\triangle_{6}(n)=(\ldots, P, 1,1,0), \quad \triangle_{7}(n)=(\ldots, P, 1,1,1), \quad \triangle_{8}(n)=(\ldots, P, 1,0, P) .
\end{gathered}
$$

Denote

$$
\lim _{n \rightarrow \infty} \frac{\triangle_{1}(n)}{\triangle_{1}(n)+\triangle_{2}(n)}=\gamma, \quad \lim _{n \rightarrow \infty} \frac{\triangle_{4}(n)}{\triangle_{4}(n)+\triangle_{5}(n)}=\alpha, \quad \lim _{n \rightarrow \infty} \frac{\triangle_{6}(n)}{\triangle_{6}(n)+\triangle_{7}(n)}=\nu
$$

Then the matrix $\mathcal{L}^{(3)}(\beta)$ has the following form

$$
\mathcal{L}^{(3)}=\left(\begin{array}{ccccc}
0 & 0 & 1 & 0 & 0 \\
\nu^{\beta} & (1-\nu)^{\beta} & 0 & 0 & 0 \\
0 & 0 & 0 & \alpha^{\beta} & (1-\alpha)^{\beta} \\
0 & 0 & 1 & 0 & 0 \\
\gamma^{\beta} & (1-\gamma)^{\beta} & 0 & 0 & 0
\end{array}\right) .
$$

Here the first column (and row) correspond respectively to $f(0,1), f(1,1), f(P, 0), f(0, P)$, and $f(1, P)$. Notice again that this system of approximations is exact in the limit $k \rightarrow \infty$. 


\begin{tabular}{|l||c|c|c|c|c|}
\hline \multicolumn{1}{|l||}{} & \multicolumn{2}{c|}{ Standard Map } & \multicolumn{2}{c|}{ Dimensions for Circle Maps } \\
\hline $\begin{array}{l}\text { Rotation } \\
\text { Number } \rho\end{array}$ & $\begin{array}{c}\text { Critical } \\
\text { Parameter }\end{array}$ & Dimension & Sine Map & $\begin{array}{c}\text { Break Map } \\
c=2\end{array}$ & $\begin{array}{c}\text { Break Map } \\
c=5\end{array}$ \\
\hline \hline$[1,1,1, \ldots]$ & 0.971635 & 0.9893 & 0.9216 & 0.9852 & 0.9236 \\
\hline$[2,2,2, \ldots]$ & 0.957445 & 0.9872 & 0.9165 & 0.9840 & 0.9170 \\
\hline$[3,3,3, \ldots]$ & 0.890863 & 0.9823 & 0.9066 & 0.9814 & 0.9029 \\
\hline$[4,4,4, \ldots]$ & 0.804725 & 0.9750 & 0.8940 & 0.9776 & 0.8821 \\
\hline \hline$[1,2,1,2, \ldots]$ & 0.876067 & 0.9808 & 0.9134 & 0.9807 & 0.8912 \\
\hline$[2,1,2,1, \ldots]$ & 0.940283 & 0.9808 & 0.9134 & 0.9844 & 0.9278 \\
\hline$[1,3,1,3, \ldots]$ & 0.772375 & 0.9672 & 0.9012 & 0.9736 & 0.8482 \\
\hline$[3,1,3,1, \ldots]$ & 0.845313 & 0.9672 & 0.9012 & 0.9817 & 0.9222 \\
\hline$[1,4,1,4, \ldots]$ & 0.684209 & 0.9530 & 0.8889 & 0.9647 & 0.8007 \\
\hline$[4,1,4,1, \ldots]$ & 0.751106 & 0.9530 & 0.8889 & 0.9784 & 0.9147 \\
\hline$[2,3,2,3, \ldots]$ & 0.920194 & 0.9821 & 0.9099 & 0.9806 & 0.8920 \\
\hline$[3,2,3,2, \ldots]$ & 0.898046 & 0.9821 & 0.9099 & 0.9834 & 0.9204 \\
\hline$[2,4,2,4, \ldots]$ & 0.873408 & 0.9732 & 0.9008 & 0.9753 & 0.8584 \\
\hline$[4,2,4,2, \ldots]$ & 0.811209 & 0.9732 & 0.9008 & 0.9817 & 0.9183 \\
\hline$[3,4,3,4, \ldots]$ & 0.867902 & 0.9772 & 0.8996 & 0.9776 & 0.8759 \\
\hline$[4,3,4,3, \ldots]$ & 0.815964 & 0.9772 & 0.8996 & 0.9806 & 0.9051 \\
\hline \hline$[1,1,2,1,1,2, \ldots]$ & 0.957041 & 0.9869 & 0.9170 & 0.9841 & 0.9172 \\
\hline$[1,1,3,1,1,3, \ldots]$ & 0.915727 & 0.9813 & 0.9082 & 0.9815 & 0.9030 \\
\hline$[1,2,2,1,2,2, \ldots]$ & 0.906712 & 0.9859 & 0.9151 & 0.9836 & 0.9146 \\
\hline$[1,2,3,1,2,3, \ldots]$ & 0.887467 & 0.9814 & 0.9082 & 0.9815 & 0.9035 \\
\hline$[1,3,2,1,3,2, \ldots]$ & 0.824204 & 0.9811 & 0.9084 & 0.9815 & 0.9035 \\
\hline$[1,3,3,1,3,3, \ldots]$ & 0.815351 & 0.9777 & 0.9033 & 0.9800 & 0.8953 \\
\hline$[2,2,3,2,2,3, \ldots]$ & 0.945871 & 0.9843 & 0.9118 & 0.9827 & 0.9098 \\
\hline$[2,3,3,2,3,3, \ldots]$ & 0.919487 & 0.9827 & 0.9086 & 0.9819 & 0.9052 \\
\hline
\end{tabular}

Table 1: Dimension of the invariant measure on the critical curve computed for a variety of rotation numbers. 


\section{$3 \quad$ Numerical Results}

Table 1 displays the results of the dimension calculations for various rotation numbers. All dimensions were computed initially using a direct approximation to the information dimension as described in Section 2.1, and corroborated by computing the transfer-matrix operator as described in Section 2.2 for large $n$ and $k$. We believe all numbers to be accurate to within 1 in the last decimal place shown. We computed dimensions for several other rotation numbers whose continued fraction expansions are eventually periodic with the periodic part matching one of the above rotation numbers. In all cases, the computed dimension was identical to the dimension in the above table to the number of decimal places shown. We also repeated the computations for the standard and sine maps with $\sin 2 \pi x$ replaced by $(1 / 2) \sin 2 \pi x+(1 / 4) \sin 4 \pi x$ and again the results matched those in Table 1.

Notice though that for the map with the break singularity, rotation numbers with period 2 expansions that eventually have the same periodic pattern but are "out of phase" yield different dimensions. The reason for this phenomenon is the asymmetry of the map at the singular point. For a few rotation numbers, we computed the dimension for the break map for several more values of the parameter $c$, including some very large ones. The results are shown in Table 2. For the larger values of $c$, the parameter interval on which the rotation number is nonzero is very small, and thus the rotation number depends sensitively on the parameter in this interval. This results in a loss of precision in the data as $c$ increases.

\begin{tabular}{|r||c|c|c|}
\hline \multicolumn{1}{|c||}{} & \multicolumn{3}{c|}{ Dimensions for the Break Map } \\
\hline Parameter $c$ & $\rho=[1,1,1, \ldots]$ & $\rho=[2,2,2, \ldots]$ & $\rho=[3,1,3,1, \ldots]$ \\
\hline \hline 1 & 1.0000 & 1.0000 & 1.0000 \\
\hline 2 & 0.9852 & 0.9840 & 0.9736 \\
\hline 3 & 0.9634 & 0.9603 & 0.9297 \\
\hline 5 & 0.9236 & 0.9170 & 0.8482 \\
\hline 10 & 0.8533 & 0.8409 & 0.720 \\
\hline 100 & 0.603 & 0.584 & 0.42 \\
\hline 1000 & 0.435 & 0.43 & 0.28 \\
\hline 10000 & 0.33 & 0.33 & 0.2 \\
\hline
\end{tabular}

Table 2: Dimension of the invariant measure computed for various values of the parameter characterizing a break singularity. 


\section{Conclusions}

An important observation we can make immediately is that in all cases, the golden mean gives the largest dimension among all rotation numbers. It has long been known numerically that for the standard map the golden mean KAM curve is the most stable, in the sense that it is the last invariant curve to be destroyed as the parameter $k$ is increased. Our results give support to this conjecture, and offer another sense in which the golden mean critical curve is exceptional. Notice also that for the standard map smaller dimensions tend to correspond to smaller critical parameter values. However, a precise formulation of this relationship is difficult because the critical parameter depends on the entire continued fraction expansion of the rotation number $\rho$, not just the eventual periodic part.

Next, notice that generally speaking the dimension decays with the growth of the entries in the continued fraction expansion of $\rho$, though one can find exceptions in Table 1 . In particular, periodic expansions whose entries are nearly equal often give larger dimensions than expansions with smaller but not as close to each other. For example, the rotation

number $[3,4,3,4, \ldots]$ give a larger dimension than $[1,1,4,1,4, \ldots]$ in all of the cases we studied, and larger even than $[1,3,1,3, \ldots]$ in many of the cases. We further observe that the ordering of rotation numbers from highest to lowest dimension is similar for the different types of maps but not precisely the same.

Finally, notice that the dimensions in Table 1 are close to one. We have two remarks concerning this. First, the dimensions decay with the growth of the strength of the singularity. For instance, we find both theoretically and numerically that the dimension tends to zero like const./ $\log c$ as the parameter $c$ for the break map tends to infinity, as shown in Table 2. Second, the rotation numbers displayed in Table 1 are very special and are likely to give among the highest possible dimensions for each type of map. We do not anticipate, however, that the dimension approaches zero as one or more of the entries in a periodic continued fraction expansion approach infinity.

\section{References}

[1] R. Artuso, E. Aurell, P. Cvitanovic, Recycling of strange sets: I. Circle expansions, II. Applications, Nonlinearity 3 (1990), 325-386.

[2] E. Aurell, On the metric properties of the Feigenbaum Attractor, J. Stat. Phys. 47 (1987), 439-458. 
[3] J. Balatoni \& A. Rényi, Remarks on entropy, Publ. Math. Inst. Hung. Acad. Sci. 1 (1956), 9-40 (in Hungarian with English summary); English translation in Selected Papers of Alfréd Rényi, vol. 1, Akadémiai Kiadó, Budapest (1976), 558-586.

[4] G. D. Birkhoff, Sur quelques courbes fermees remarquable, Bull. Soc. Math. France 60 (1932); also in Collected Papers, vol. II, AMS (1950), 444-461.

[5] P. Collet, J. L. Lebowitz, \& A. Porzio, The dimension spectrum of some dynamical systems, J. Stat. Phys. 47 (1987), 609-644.

[6] J. D. Farmer, E. Ott, \& J. A. Yorke, The dimension of chaotic attractors, Physica 7D (1983), 153-180.

[7] M. J. Feigenbaum, Quantitative universality for a class of nonlinear transformations, J. Stat. Phys. 19 (1978), 25-52.

[8] M. J. Feigenbaum, The universal metric properties of nonlinear transformations, J. Stat. Phys. 21 (1979), 669-706.

[9] M. J. Feigenbaum, L. P. Kadanoff, S. J. Shenker, Quasiperiodicity in dissipative systems: a renormalization group analysis, Physica 5D (1982), 370-386.

[10] J. M. Greene, A method for determining a stochastic transition, J. Math. Phys. 20 (1979), 1183-1201.

[11] J. Graczyk \& G. Świạtek, Critical circle maps near bifurcation, SUNY Stony Brook IMS preprint \#1991/8.

[12] J. Graczyk \& G. Świạtek, Singular measures in circle dynamics, Comm. Math. Phys. 157 (1993), 213-230.

[13] T. Halsey, M. Jensen, L. Kadanoff, I. Procaccia, B. Shraiman, Fractal measures and their singularities: the characterization of strange sets, Phys. Rev. A 33 (1986), 1141-1151.

[14] M. Herman, Sur la conjugasion différentiable des difféomorphismes du cercle à des rotations, Pub. Math. IHES 49 (1979), 5-233.

[15] M. Herman, Sur les Courbes Invariantes par les Difféomorphismes de l'Anneau, vol. 1, Astérisque 103-104 (1983).

[16] Y. Katznelson \& D. Ornstein, The differentiability of the conjugation of certain diffeomorphisms of the circle, Erg. Th. Dyn. Sys. 9 (1989), 643-680. 
[17] Y. Katznelson \& D. Ornstein, A new method for twist theorems, J. d'Analyse 60 (1993), $157-208$.

[18] K. M. Khanin, Universal estimates for critical circle mappings, Chaos 1 (1991), 181-186.

[19] K. M. Khanin, Rigidity for circle homeomorphisms with the break singularity, Russian Acad. Sci. Dokl. Math. (to appear).

[20] K. M. Khanin \& E. B. Vul, Circle homeomorphisms with weak discontinuities, Adv. Soviet Math. 3 (1991), 57-98.

[21] R. S. Mackay, Renormalization in Area-Preserving Maps, World Scientific, Singapore (1993).

[22] A. Osbaldestin \& M. Sarkis, Singularity spectrum of a critical KAM torus, J. Phys. A: Math. Gen. 20 (1987), L953-958.

[23] S. Ostlund, D. Rand, J. Sethna, E. Siggia, Universal properties of the transition from quasi-periodicity to chaos in dissipative systems, Physica 8D (1983), 303-342.

[24] Ya. G. Sinai, Topics in Ergodic Theory, Princeton University Press, Princeton, NJ (1994).

[25] A. Stirnemann, Renormalization for Golden Circles, Comm. Math. Phys. 152 (1993), 369-431.

[26] D. Sullivan, private communication.

[27] G. Świạtek, Rational rotation numbers for maps of the circle, Comm. Math. Phys. 119 (1988), 109-128.

[28] E. B. Vul, Ya. G. Sinai, \& K. M. Khanin, Feigenbaum universality and the thermodynamic formalism, Russ. Math. Surv. 39:3 (1984), 1.

[29] L.-S. Young, Dimension, entropy and Lyapunov exponents, Erg. Th. Dyn. Sys. 2 (1982), 109-124. 